\title{
Changes in mesenchymal stem cells following long-term culture in vitro
}

\author{
YAJUN GU ${ }^{1,2^{*}},{\text { TAO } \mathrm{LI}^{2 *}, \text { YANLING DING }}^{2}$, LINGXIAN SUN ${ }^{2}$, \\ TAO TU ${ }^{2}$, WEI ZHU ${ }^{2}$, JIABO HU ${ }^{2}$ and XIAOCHUN SUN ${ }^{2}$ \\ ${ }^{1}$ The Blood Bank of Jiangyin, Wuxi, Jiangsu 214400; ${ }^{2}$ School of Medical Science and Laboratory Medicine, \\ Jiangsu University, Zhenjiang, Jiangsu 212013, P.R. China
}

Received May 22, 2015; Accepted February 22, 2016

DOI: $10.3892 / \mathrm{mmr} .2016 .5169$

\begin{abstract}
Mesenchymal stem cells (MSCs), which can be isolated from umbilical cords and induced to differentiate into multiple cell types in vitro, represent an ideal source for cell and gene therapy. MSCs are typically expanded in culture prior to their therapeutic application. However, similar to other types of stem cell, MSCs undergo senescence following a certain number of cell expansion passages in vitro, and eventually stop proliferating. The objective of the present study was to measure the changes that occur over successive passages of MSCs during long-term in vitro culture, and to detect the effect of aging on MSC morphology, phenotype, proliferation, cell cycle, differentiation, intracellular reactive oxygen species (ROS) levels and gene expression. To understand the importance of oxidative stress in the aging of adult stem cells, the current study established a cell model of $\mathrm{H}_{2} \mathrm{O}_{2}$-induced MSC premature senescence. Analysis of the biological characteristics of human umbilical cord MSCs during replicative and premature senescence revealed the importance of extrinsic factors in the aging of stem cells, particularly ROS. The findings of the present study suggest that cellular senescence, a state of irreversible growth arrest, can be triggered by ROS. Thus, it is important to improve the extrinsic culture environment of MSCs to retain the phenotype of expanded cells and delay the process of senescence prior to their clinical application.
\end{abstract}

\section{Introduction}

Mesenchymal stem cells (MSCs) are a promising cell source for use in tissue regeneration and their potential therapeutic

Correspondence to: Professor Xiaochun Sun, School of Medical Science and Laboratory Medicine, Jiangsu University, 301 Xuefu Road, Zhenjiang, Jiangsu 212013, P.R. China

E-mail: xiaochun@ujs.edu.cn

${ }^{*}$ Contributed equally

Key words: mesenchymal stem cells, senescence, reactive oxygen species, premature senescence, $\mathrm{H}_{2} \mathrm{O}_{2}$ application has already been investigated in several clinical trials (1-3). Human MSCs have the ability to differentiate into different mesodermal cell lineages, including osteocytes, chondrocytes, adipocytes, hepatocytes and neurons $(4,5)$. Therefore, MSCs have important therapeutic potential. Typically, these cells are expanded prior to their clinical application. However, MSCs have a limited lifespan in vitro, as do any normal somatic cells, and there is no precise molecular definition of MSC long-term culture in vitro. Several studies have demonstrated that long-term culture of MSCs results in continuous changes to the cells, including decreased proliferation rate, increased cell size and different differentiation potentials $(6,7)$. These problems have hindered the expansion of MSCs for therapeutic use, causing a major bottleneck in clinical applications. MSCs have become an attractive therapeutic tool due of their unique characteristics, including their ability to self-renewal, and ease of isolation and expansion. MSCs possess a broad spectrum for regenerative medicine due to their potential to repair tissue and to differentiate into osteoblasts, chondroblasts, adipocytes and myoblasts. The transplantation of pluripotent MSCs has been tested in the treatment of neurological disorders, such as Parkinson's disease, cerebral infarction, brain injury and spinal cord injury, bone tissue engineering, cardiovascular diseases, severe liver damage repair, pulmonary fibrosis and reduction of bone marrow transplant rejection indicating the potential for porting applications.

It has been reported that long-term culture of MSCs causes the cells to undergo replicative senescence with the cell morphology becoming enlarged and flattened, the development of prominent nucleoli and cytoplasmic granules, alteration in the differentiation potential, and shortened telomere length over progressively increasing passages (8). Additionally, these senescent cells can be stained by senescence-associated $\beta$-galactosidase (SA- $\beta$-gal). It is important, therefore, to fully understand the biological alterations that occur in these expanded stem cell populations.

Cellular senescence is induced by intrinsic and extrinsic factors $(9,10)$. With numerous passages, cell senescence can be triggered by replicative exhaustion, DNA damage and telomere shortening. Increasing evidence indicates that the continuous accumulation of intracellular reactive oxygen species (ROS) is a major initiating factor of replicative senescence $(11,12)$. Furthermore, extrinsic stresses, such as oxidative stress, may 
affect intrinsic factors, and subsequently lead to DNA damage accumulation and telomere shortening. In addition to replicative senescence, premature senescence is another model of in vitro senescence.Premature senescence is induced by various extrinsic factors, including hydrogen peroxide, ionizing radiation, high glucose, D-galactose, high oxygen and old rat serum (13-15). In addition, human fibroblasts undergo premature senescence when cultured in 8-methoxypsoralen/ultraviolet A conditions (16). Zhang et al (14) reported that old rat serum induced the senescence of adult MSCs, and inhibited their proliferation and survival. Apurinic/apyrimidinic endonuclease 1/redox factor-1 (APE1/Ref-1) is a redox factor for transcription factors that alters trinucleotide stores, which are vital to energy metabolism, via regulation of DNA repair processes and transcription factors, including P53 (17). P21, a cyclin-dependent kinase inhibitor, is a major transcriptional target of P53. It was originally identified as a gene that inhibits DNA synthesis and promotes cell cycle arrest (18). Further analysis demonstrated that senescence of numerous cell lines was correlated with the upregulation of P21 and P53 (19). Thus, establishing an optimized microenvironment for the culture and expansion of MSCs that preserves their properties and prolongs their lifespan is imperative (20-23).

Cellular senescence is a complex process and the sequence of its molecular mechanisms is thus far unknown. Sharpless and DePinho (24) proposed that humans grow old due to stem cell aging as a result of mechanisms designed to suppress the development of cancer. Thus, it may be important to determine how individuals grow old and how cancer develops via investigation of the process of stem cell aging. The current study compares several biological characteristics of human umbilical cord MSCs (hucMSCs) during long-term in vitro expansion for passages 4, 11 and 17 (P4, P11 and P17). In addition, the present study established an $\mathrm{H}_{2} \mathrm{O}_{2}$-induced premature senescence model to examine MSC senescence.

\section{Materials and methods}

Isolation and culture of hucMSCs. The experimental protocol was approved by Jiangsu University ethics committee (Zhenjiang, China). Fresh umbilical cords were collected in March 2015, immediately after birth from healthy donors at the First People's Hospital of Zhenjiang (Zhenjiang, China). The umbilical cords were rinsed twice in phosphate-buffered saline (PBS) until the cord blood was cleared. The blood vessels were removed from each cord, then the remaining tissue was cut into $1-\mathrm{mm}^{3}$ pieces, and suspended in low-glucose Dulbecco's modified Eagle's medium (L-DMEM; Gibco; Thermo Fisher Scientific, Inc., Waltham, MA, USA) containing $10 \%$ fetal bovine serum (FBS; Thermo Fisher Scientific, Inc.), 1\% penicillin and $1 \%$ streptomycin (Beyotime Institute of Biotechnology, Haimen, China). All cultures were incubated at $37^{\circ} \mathrm{C}$ with an atmosphere of $5 \% \mathrm{CO}_{2}$ in a humidified chamber. The medium was changed every 3 days after initial plating. When well-developed colonies of fibroblast-like cells reached $70-80 \%$ confluence, the cells were trypsinized with $0.25 \%$ trypsin-EDTA (Thermo Fisher Scientific, Inc.) and passaged into new culture flasks for further expansion. To establish a cell model of $\mathrm{H}_{2} \mathrm{O}_{2}$-induced MSC premature senescence, early passage (P4) hucMSCs were treated with $\mathrm{H}_{2} \mathrm{O}_{2}$ at different concentrations $(0,200,400,600$ and $800 \mu \mathrm{M})$ for $2 \mathrm{~h}$. The stimulus was then removed and cells were further incubated in fresh medium for $48 \mathrm{~h}$.

Flow cytometry. To determine the phenotypes of hucMSCs, fluorescein isothiocyanate (FITC)- or phycoerythrin (PE)-labeled mouse monoclonal antibodies against human leukocyte antigen (HLA)-DR, cluster of differentiation (CD)105, CD34, CD29, CD90 and CD44 (1:10; BD Biosciences, Franklin Lakes, NJ, USA; cat. nos. 555811, 560839, 348053, 555443, 555596 and 555479, respectively) were used. Briefly, at P4, MSCs were trypsinized, washed twice with PBS and stained with the monoclonal antibodies, according to the manufacturer's protocol. Mouse monoclonal PE-immunoglobulin G1 (IgG1) and FITC-IgG1 (BD Biosciences; cat. nos. 555574 and 555573, respectively) were used as isotype controls. The stained cells were analyzed using the FACSAria flow cytometer(BD Biosciences).

Morphological observation of hucMSCs. The cell surface morphology of hucMSCs was analyzed using scanning electron microscopy (SEM; Hitachi S-3400N; Hitachi, Ltd., Tokyo, Japan) at P4, P11 and P17. Cells were seeded in 6-well plates and the media was removed following 3 days of culture. The cells were washed with PBS and fixed with $2.5 \%$ glutaraldehyde (Sangon Biotech Co., Ltd., Shanghai, China) for $1 \mathrm{~h}$. The cells were then rinsed with distilled water and dehydrated with a series of ethanol gradients starting at $30 \%$ and increasing to $50,70,80,90,95$ and $100 \%$ (v/v). Subsequently, the cells were air-dried overnight at room temperature in a fume hood. The cells were gold-coated and cell morphology was analyzed using SEM.

To image the cell nuclei, cells were cultured at a comparable density on coverslips in 6-well plates. They were washed with PBS, fixed for 15 min with $4 \%$ formaldehyde (Sangon Biotech Co., Ltd.) and washed with PBS 3 times. DNA was visualized with 4'6-diamidino-2-phenylindole $(1 \mathrm{mg} / \mathrm{ml})$ (Beyotime Institute of Biotechnology) by fluorescence microscopy (Molecular Devices, LLC, Sunnyvale, CA, USA).

Cell proliferation assay. Assessment of the proliferative ability of hucMSCs was performed using a 3-(4,5-dimethylthiazol-2-yl)-2,5-diphenyl-2-H-tetrazolium bromide (MTT; AMRESCO LLC, Solon, OH, USA) assay at P4, P11 and P17. The cells were seeded in 96-well plates at a density of 1,000 cells per well. At days 1, 2, 3, 4, 5, 6 and 7, MTT $(20 \mu \mathrm{l})$ was added to each well for $4 \mathrm{~h}$. When the reaction was terminated, the medium was discarded and $150 \mu \mathrm{l}$ dimethylsulfoxide (Sigma-Aldrich, St. Louis, MO, USA) was added to each well. Following uniform oscillation for $10 \mathrm{~min}$ to fully dissolve the purple formazan crystals, the absorbance values were determined at $490 \mathrm{~nm}$ with a spectrophotometer (FLX800; BioTek Instruments, Inc., Winooski, VT, USA).

SA- $\beta$-gal staining. SA- $\beta$-gal activity was analyzed in different passages (P4, P11 and P17) of hucMSCs using a SA- $\beta$-gal staining kit (Beyotime Institute of Biotechnology), according to the manufacturer's protocol. In brief, the cells were cultured to comparable densities on coverslips in 24-well plates and washed with PBS, fixed for 15 min with $4 \%$ formaldehyde 
and washed with PBS. Subsequently, the cells were incubated overnight at $37^{\circ} \mathrm{C}$ in a $\mathrm{CO}_{2}$-free chamber with freshly prepared SA- $\beta$-gal stain solution. SA- $\beta$-gal-positive cells exhibited a blue color; the number of positive cells was counted for every 200 cells in randomly selected fields of view using light microscopy (TE300; Nikon Corporation, Tokyo, Japan).

Cell cycle analysis. Cells at P4, P11 and P17 were collected, washed twice with PBS and stained with propidium iodide (Sigma-Aldrich) for $30 \mathrm{~min}$ in dark conditions. The stained cells were analyzed by flow cytometry (FACSAria; BD Biosciences).

Generation of conditioned media (CM) for hucMSC migration assay. To prepare $\mathrm{CM}, 8 \times 10^{4}$ hucMSCs of $\mathrm{P} 4, \mathrm{P} 11$ and $\mathrm{P} 17$ were plated on 6-well culture plates with $10 \%$ FBS L-DMEM and allowed to adhere overnight at $37^{\circ} \mathrm{C}$ with $5 \% \mathrm{CO}_{2}$ atmosphere. The following day, the media was removed, the cells were washed twice with PBS and then re-incubated with $1.5 \mathrm{ml}$ serum-free culture media. After $12 \mathrm{~h}$, the CM was collected, centrifuged for $5 \mathrm{~min}$ at $447 \mathrm{x} \mathrm{g}$ to remove cell debris and passed through a $0.45-\mu \mathrm{m}$ filter (Sigma-Aldrich). CM aliquots were frozen at $-20^{\circ} \mathrm{C}$ until analysis (not exceeding 2 weeks).

P4 MSCs $\left(4 \times 10^{4}\right)$ in $200 \mu 1$ serum-free L-DMEM were plated in the upper chambers and $600 \mu$ l undiluted CM with $10 \%$ FBS was added to the lower chambers. After $10 \mathrm{~h}$ of incubation, the cells were fixed with $4 \%$ formaldehyde for $30 \mathrm{~min}$. The cells that remained on the membrane of the upper chamber were removed with cotton swabs and migrating cells were stained with crystal violet (Sigma-Aldrich). Four low-power fields (x100) were randomly selected in each chamber to observe the cells and the number of stained migrated cells on each image was counted.

ROS detection. To detect the accumulation of intracellular ROS in hucMSCs, a ROS assay kit (Beyotime Institute of Biotechnology) was used. Following culture to different passages (P4, P11 and P17), the cells were washed 3 times in serum-free medium and incubated in a final concentration of $10 \mathrm{mM}$ dihydrodichlorofluorescein diacetate (H2DCFDA) at $37^{\circ} \mathrm{C}$ for $25 \mathrm{~min}$. The media was then removed and cells were washed 3 times with serum-free medium. The cells were observed using a fluorescence microscope (TE300; Nikon Corporation).

To quantify the ROS level, the H2DCFDA fluorescence intensity of the cells was detected by flow cytometry. Briefly, $5 \times 10^{4}$ cells were collected and resuspended in a final concentration of $10 \mathrm{mM} \mathrm{H} 2 \mathrm{DCFDA}$ with serum-free medium. After $25 \mathrm{~min}$ incubation at $37^{\circ} \mathrm{C}$, cells were washed with serum-free medium 3 times and resuspended in PBS, then placed on ice for immediate detection using a FACScan flow cytometer (BD Biosciences) with excitation at $488 \mathrm{~nm}$ and emission at $525 \mathrm{~nm}$.

HucMSC differentiation assays. HucMSCs at P4, P11 and P17 were cultured in a medium containing either osteogenic (50 $\mathrm{mM}$ ascorbate-phosphate, $10 \mathrm{mM} \beta$-glycerophosphate and $0.1 \mathrm{mM}$ dexamethasone) or adipogenic (1 mM dexamethasone, $10 \mathrm{mM}$ insulin, $0.5 \mathrm{mM}$ isobutyl-methylxanthine and $200 \mathrm{mM}$ indomethacin) reagents (Sigma-Aldrich) at $5 \% \mathrm{CO}_{2}$ at $37^{\circ}$.
After 2 weeks of culturing, osteogenic differentiation was assessed by the examination of neutrophil alkaline phosphatase with Alizarin Red dye (Sigma-Aldrich). After 3 weeks of culturing, adipogenic differentiation was detected via intracellular lipid vesicles; the cells were stained with Oil Red-O (Cyagen, Guangzhou, China) to detect lipids using an inverted microscope (TE300; Nikon Corporation).

Reverse transcription-polymerase chain reaction (RT-PCR). Total RNA was isolated using TRIzol reagent (Invitrogen; Thermo Fisher Scientific, Inc.), according to the manufacturer's instructions. Reverse transcription was conducted using Moloney murine leukemia virus reverse transcriptase (Promega, USA) and the obtained cDNA was subjected to PCR. PCR was performed using $1 \mu \mathrm{g}$ of cDNA sample with $0.3 \mathrm{U}$ of Taq polymerase (CinnaGen Co., Tehran, Iran), $200 \mu \mathrm{M}$ dNTPs, $10 \mathrm{pM}$ of each primer, reaction buffer and $\mathrm{MgCl} 2$ (Takara Bio, Inc., Otsu, Japan) in a $25-\mu 1$ volume. PCR amplification was performed for 35 cycles using an ABI 2720 thermal cycler (Applied Biosystems; Thermo Fisher Scientific, Inc.). The cycling conditions were: $94^{\circ} \mathrm{C}$ for $30 \mathrm{sec}, 60^{\circ} \mathrm{C}$ (primer) for $30 \mathrm{sec}, 72^{\circ} \mathrm{C}$ for $30 \mathrm{sec}$ and a final extension at $72^{\circ} \mathrm{C}$ for $10 \mathrm{~min}$. The PCR products were separated on a $1.5 \%$ agarose gel, stained with ethidium bromide (Thermo Fisher Scientific, Inc.) and visualized under UV light using the GeneGenius bio-imaging system (Syngene, MD, USA). Primers were as follows: APE1/Ref-1, F 5'-GCTTCGAGCCTGGATTAAGA-3' and R 5'-TCATCGCCTATGCCGTAAGA-3'; P21, F 5'-CTA CCTCAGGCAGCTCAAG-3' and R, 5'-AGCCTCTACTGC CACCATC-3'; P53, F 5'-TCTGTGACTTGCACGTACTC-3' and R 5'-TGTAGTGGATGGTGGTACAG-3'; $\beta$-actin, F 5'-CACGAAACTACCTTCAACTC-3' and R 5'-CATACT CCTGCTTGCTGATC-3'. The primers were produced by Shanghai Bio-Engineering Company (Shanghai, China).

Statistical analysis. All data are expressed as the mean \pm standard deviation. Statistical analysis was performed using GraphPad Prism software (version 5; GraphPad Software, Inc., La Jolla, CA, USA). All experiments were replicated 3 times. Analysis of variance was used to analyze variance among all groups, and Student's t-test was performed to compare experimental and relative control groups. $\mathrm{P}<0.05$ was considered to indicate a statistically significant difference.

\section{Results}

Morphology and cell surface characteristics of hucMSCs in primary culture. Following the initial 7 days of primary culture, hucMSCs adhered to the plastic surface of the culture flasks and presented as a small population of single cells with spindle-like shape. On day 10 after initial plating, the cells exhibited long spindle-shaped fibroblastic cells, they had began to form colonies and were confluent (Fig. 1A). At P4, hucMSCs were positively stained with CD29, CD90, CD44 and CD105, but were negative for the hematopoietic lineage markers CD34 and HLA-DR, as measured by fluorescence-activated cell sorting (FACS) analysis (Fig. 1B).

Changes in hucMSCs during long-term in vitro culture. HucMSCs from the same donors were characterized by their 

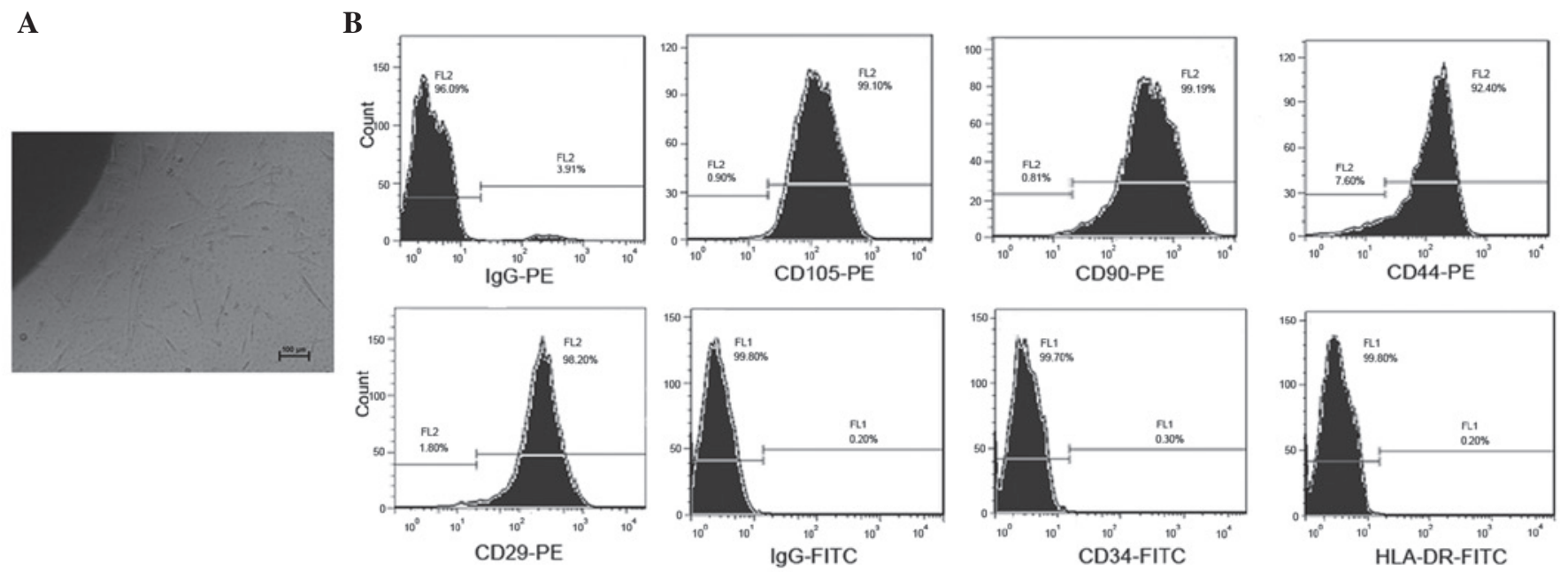

Figure 1. Isolation and identification of human umbilical cord mesenchymal stem cells (hucMSCs). (A) Primary cells at passage 0 (magnification, $\mathrm{x} 40$ ). (B) Surface markers identified by fluorescence-activated cell sorting analysis at passage 4. IgG indicates isotypes. HucMSCs are positive for CD29, CD90, CD44 and CD105, but negative for CD34 and HLA-DR. IgG, immunoglobulin G; PE, phycoerythrin; FITC, fluorescein isothiocyanate; CD, cluster of differentiation; HLA-DR, human leukocyte antigen-DR.

A

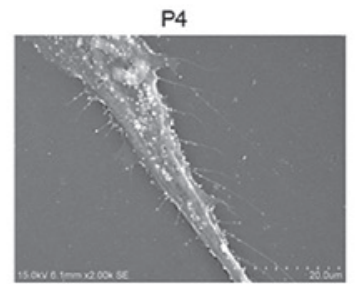

B

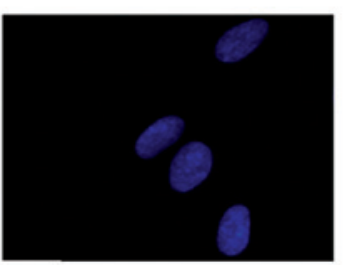

D

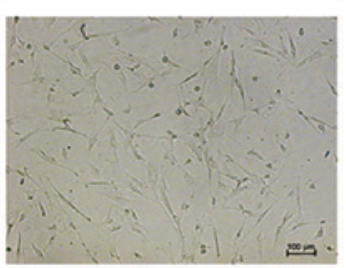

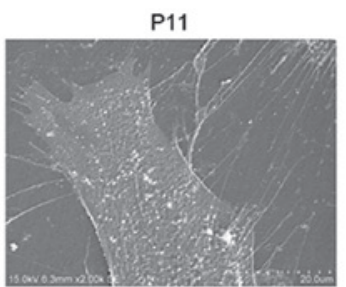
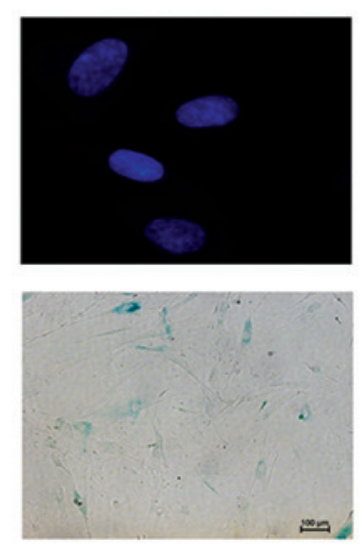
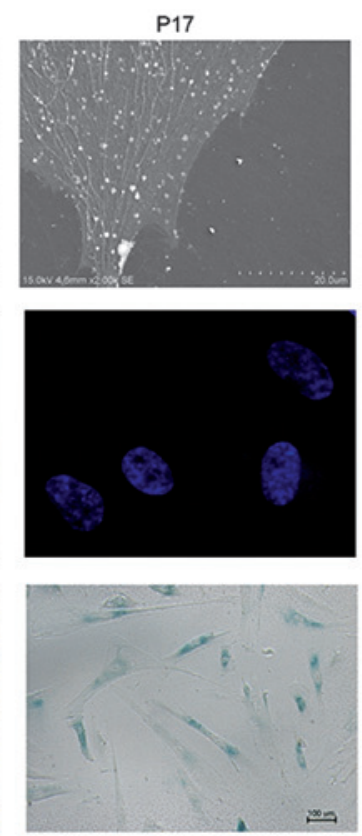

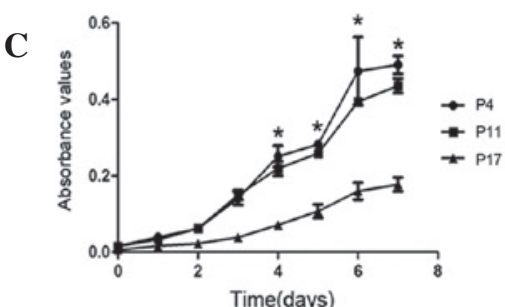

$\mathbf{E}$

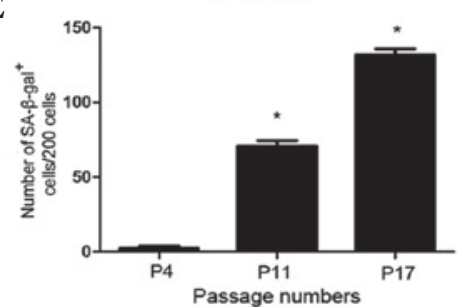

Figure 2. Characterization of long-term cultured human umbilical cord mesenchymal stem cells (hucMSCs). (A) Ultrastructure of cell membrane of long-term cultured hucMSCs detected by scanning electron microscopy (magnification, x2,000). (B) Cell nuclei visualized by 4'6-diamidino-2-phenylindole staining. Senescence-associated heterochromatic foci formation is present in the nucleus of senescent P17 hucMSCs compared with the nuclei of P4 and P11 (x200 magnification). (C) Growth curve for long-term cultured hucMSCs detected by 3-(4,5-dimethylthiazol-2-yl)-2,5-diphenyl-2-H-tetrazolium bromide assay. (D) Representative images (magnification, x100) and (E) quantification of senescent hucMSCs, as detected by SA- $\beta$-gal staining. ${ }^{*} \mathrm{P}<0.05$ vs. P4. P, passage; SA- $\beta$-gal, senescence-associated $\beta$-galactosidase.

morphology, growth, SA- $\beta$-gal activity and differentiation capacity. The present study observed morphological changes during long-term culture of hucMSCs by analyzing the cell membrane and nucleus. The ultrastructure of the cell membrane was observed under SEM (Fig. 2A). Cells at P4 were spindle-like, plump, stereoscopic and exhibited a large population of uniformly distributed vimineous microvilli. However, at the middle and late phases of the culture (P11 and P17, respectively), hucMSCs became flatter, broader and had numerous large pseudopods, often with multiple secondary bifurcations. Furthermore, the number and length of microvilli was decreased during the late phases, particularly at P17. The current study also used immunofluorescence to observe changes to the cell nuclei (Fig. 2B). At P4, the chromatin distribution in the nuclei was homogeneous and the nucleus size was uniform. Compared with P4, the nuclei at P11 and P17 were swollen, and chromatin was localized into a small area termed the senescence-associated heterochromatic foci (SAHF). It was concluded that both the 

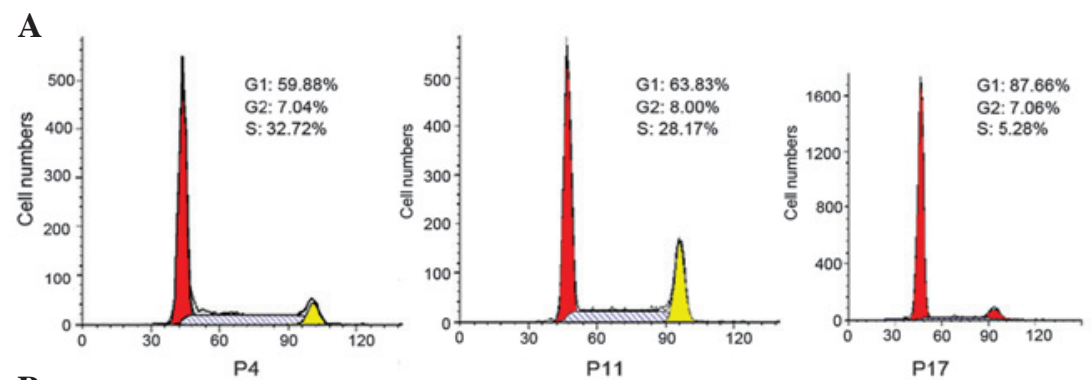

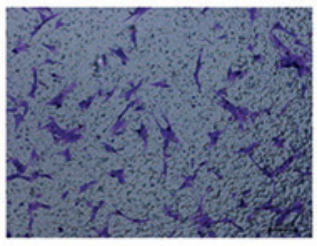

P4

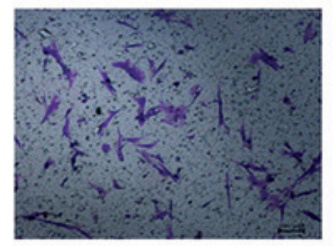

P11

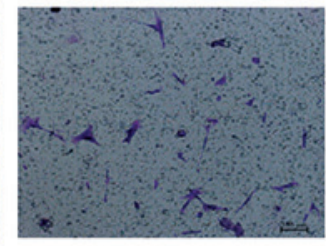

P17

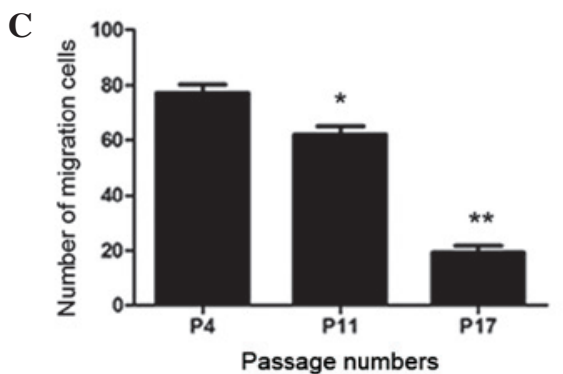

Figure 3. Effects of long-term in vitro culture on the cell cycle and microenvironment of human umbilical cord mesenchymal stem cells (hucMSCs). (A) Cell cycle distribution of hucMSCs at various passages determined by fluorescence-activated cell sorting analysis. (B) HucMSC cell migration assay using hucMSC conditioned media (crystal violet staining; magnification $\mathrm{x} 100$ ). (C) Quantification of migrated cells. ${ }^{*} \mathrm{P}<0.05$, $^{* *} \mathrm{P}<0.01 \mathrm{vs}$. $\mathrm{P} 4$. $\mathrm{n}=4$. $\mathrm{P}$, passage.
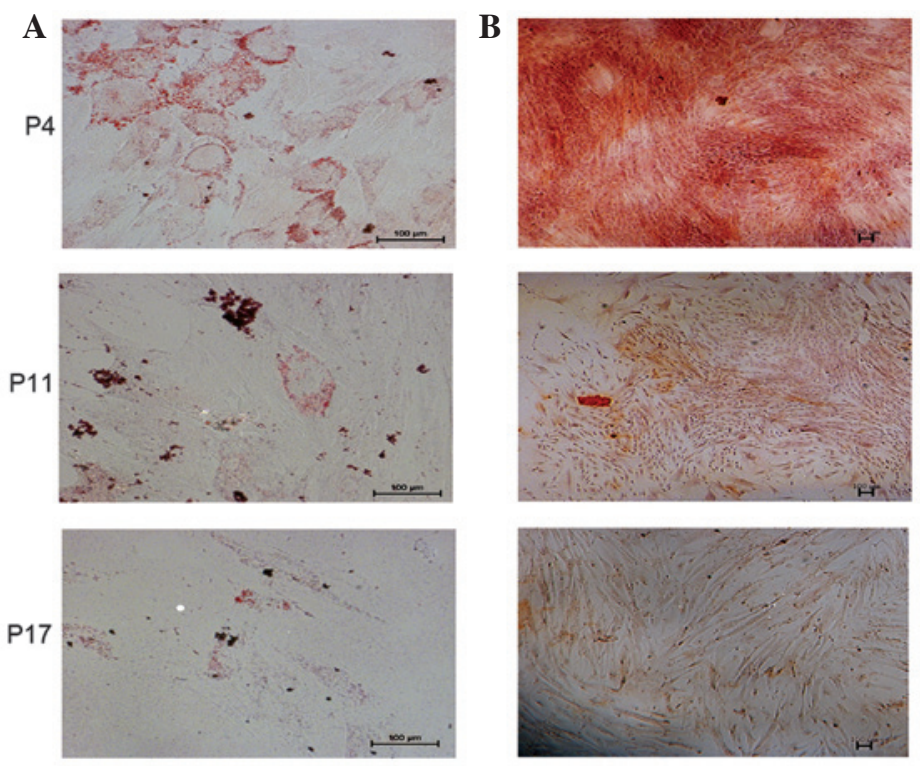

Figure 4. In vitro differentiation of human umbilical cord mesenchymal stem cells (hucMSCs). (A) HucMSCs were differentiated into adipocytes for 21 days. Fat accumulation was visualized by Oil Red-O staining (magnification, x200). (B) HucMSCs were differentiated into osteoblasts for 14 days. Osteogenic differentiation was visualized by Alizarin Red staining (magnification, x100). P, passage.

nuclei and the cell membranes of hucMSCs had undergone changes during the long-term in vitro culture.

Subsequently, the current study analyzed the growth characteristics of hucMSCs during long-term in vitro culture. The proliferation rate of hucMSCs was measured at P4, P11 and P17 by MTT assay (Fig. 2C). The hucMSC proliferation rate in the early and middle phases exhibited an S-shaped growth curve, with a minor decrease in proliferation rate in the middle phase compared with the early phase. However, when reaching $\mathrm{P} 17$, the hucMSC proliferation rate was significantly decreased compared with the early phase cells $(\mathrm{P}<0.05)$. The shape of long-term growth curves differed considerably between passages, with almost a straight line exhibited during the late phase of hucMSC culture.
Additionally, the current study detected SA- $\beta$-gal activity at the 3 passages (Fig. 2D). The number of senescent cells were increased during the middle and late phase, compared with the early phase $(\mathrm{P}<0.05$; Fig. $2 \mathrm{E})$. The results of the current study indicate that hucMSCs undergo replicative senescence during long-term in vitro culture.

Considering the functional implications of changes to growth characteristics during multiple passages, the present study analyzed the cell cycle status of hucMSCs. The cell cycle distribution at different passages was determined by FACS analysis. Single-variable histograms of DNA provided data measuring the percentages of cells in the G0/G1, S and G2/M phases of the cell cycle. As presented in Fig. 3A, hucMSCs demonstrated a progressive increase in the frequency of cells in G0/G1 phase 
A

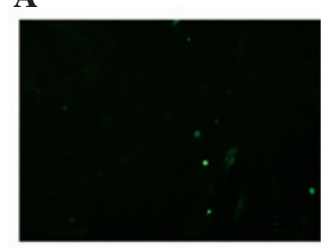

P4

C

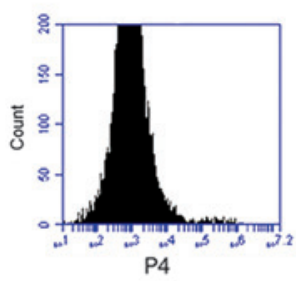

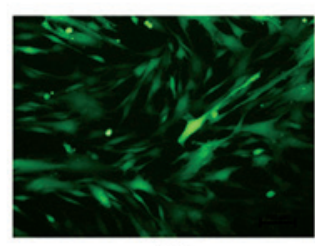

P11

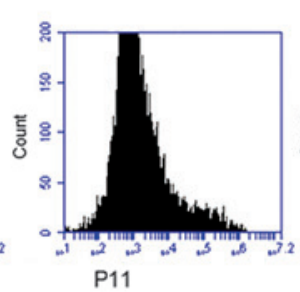

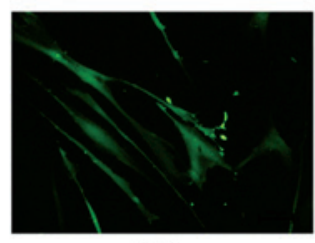

P17
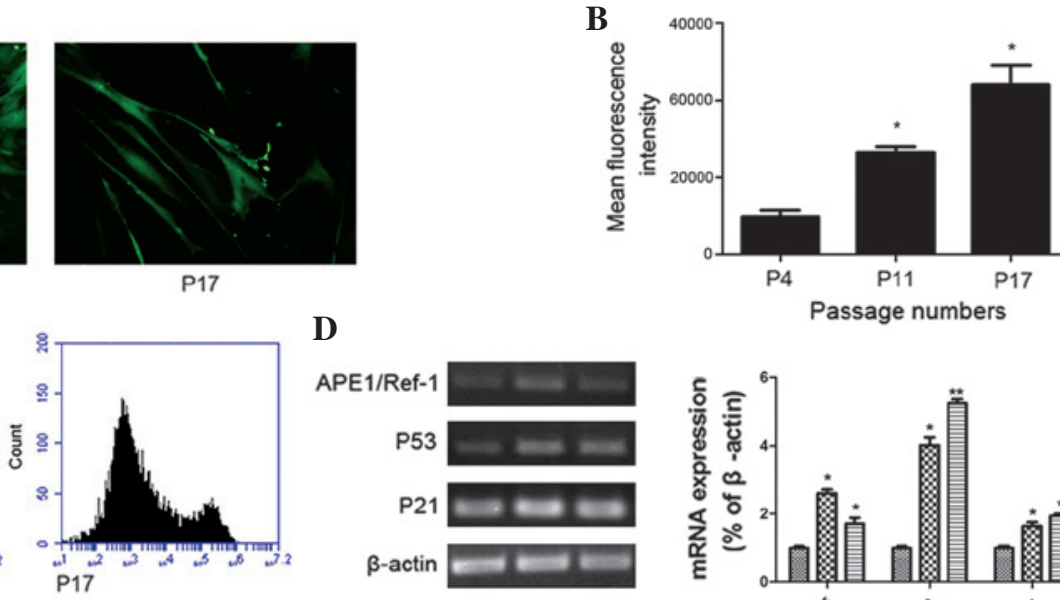

D
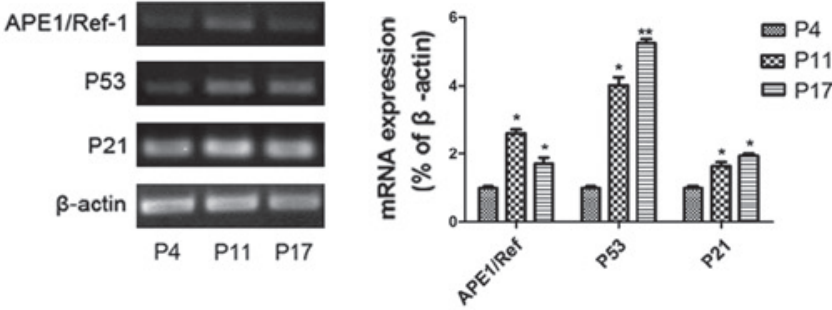

Figure 5. Effects of long-term expansion on intracellular reactive oxygen species (ROS) levels and mRNA expression in human umbilical cord mesenchymal stem cells. (A) Fluorescence microscopy identified more ROS-stained cells in the middle or late phase (P11 or P17) (dihydrodichlorofluorescein diacetate staining). (B and C) Quantification of intracellular ROS levels was performed by fluorescence-activated cell sorting (FACS) analysis of stained cells to obtain mean fluorescence intensity. ${ }^{*} \mathrm{P}<0.05$ vs. P4. (D) Reverse transcription-polymerase chain reaction analysis of the expression of P21, P53 and APE1/Ref-1. $\beta$-actin was used as an internal control. ${ }^{*} \mathrm{P}<0.05,{ }^{* *} \mathrm{P}<0.01$ vs. $\mathrm{P} 4$. P, passage; APE1/Ref-1, apurinic/apyrimidinic endonuclease $1 /$ redox factor-1.

during long-term in vitro culture. Cell cycle analysis of P17 revealed an obvious increase in the number of cells in the G0/G1 phase and a reduction of S-phase cells compared with P4 and P11. The current study did not observe the development of polyploidy in hucMSCs throughout the long-term culture.

CM was collected from P4, P11 and P17 cells, and used as the medium in the lower chamber during a cell migration assay. In the P4 and P11 CM groups, obvious migration of cells was observed, whereas few migrated cells were observed in the P17 CM group. Cell counting demonstrated that the migration of cells in the P17 CM group (19 \pm 3.4 cells/100 field) was significantly reduced compared with the P4 and P11 groups $(77 \pm 3.9$ and $62 \pm 4.3$ cells/field, respectively; $\mathrm{P}<0.01$; Fig. 3B and C). The results indicated that senescent hucMSCs may secrete toxic factors into the microenvironment that inhibit hucMSC migration and are harmful to neighboring cells.

Long-term culturing of hucMSCs impairs the capacity to differentiate in vitro. In order to observe the differentiation potential of hucMSCs at P4, P11 and P17, the cells were induced to differentiate into adipocytes or osteocytes, as measured by positive staining of Oil Red-O (Fig. 4A) and Alizarin Red (Fig. 4B), respectively. Based on visual assessment of the extent of Oil Red O-positive lipid inclusions, it was determined that the adipogenic differentiation capacity of hucMSCs was decreased at P11 and P17 compared with at P4. Similarly, the capability of osteogenic differentiation of hucMSCs was decreased at P11 and P17 compared with at P4.

Increased levels of intracellular ROS and senescence markers during the long-term culture of hucMSCs. To investigate the levels of intracellular ROS during long-term culture, a fluorescence protocol using H2DCFDA was performed on P4, P11 and P17 hucMSCs. As presented in Fig. 5A, the green fluorescence was more intense in P11 and P17 hucMSCs compared with P4 hucMSCs. This suggests that hucMSCs exhibit low intracellular ROS levels during the early phases; but the levels increase in the middle and late phases. To quantify the ROS levels, fluorescence intensity was detected by flow cytometry (Fig. 5B and C). The mean fluorescence intensity (MFI) of P11 and P17 were increased compared with at P4. The MFI was increased by 3-fold in P17 hucMSCs and by 2-fold in P11 hucMSCs compared with P4 hucMSCs. Thus, hucMSCs exhibited higher intracellular ROS levels at later passages during the long-term culture $(\mathrm{P}<0.05$; Fig. $5 \mathrm{C})$.

To further investigate the association between senescence and oxidative stress, the current study analyzed the mRNA expression of P53, P21 and APE1/Ref-1 in different phases of hucMSC culture. In agreement with a previous study (19), telomeric foci containing multiple DNA damage response factors were assembled in a subset of senescent cells and signaled through ATM to p53, upregulating p21 and causing G1 phase arrest. As demonstrated in Fig. 5D, compared with the early passages, P21, P53 and APE1/Ref-1 mRNA levels were significantly increased in the middle and late phases.

Establishing a cell model of $\mathrm{H}_{2} \mathrm{O}_{2}$-induced MSC premature senescence. Early passage hucMSCs were treated with $\mathrm{H}_{2} \mathrm{O}_{2}$ at different concentrations $(0,200,400,600$ and $800 \mu \mathrm{M})$ for $2 \mathrm{~h}$. The stimulus was then removed and cells were further incubated in fresh medium for $48 \mathrm{~h}$. The effect of $\mathrm{H}_{2} \mathrm{O}_{2}$ treatment on the cell cycle in $\mathrm{P} 4$ hucMSCs was analyzed (Fig. 6A). FACS analysis demonstrated that, with increasing concentrations of $\mathrm{H}_{2} \mathrm{O}_{2}$, the number of hucMSCs in the G0/G1 fraction progressively increased, and treatment with $600 \mu \mathrm{M} \mathrm{H}_{2} \mathrm{O}_{2}$ significantly promoted cell cycle arrest in the G0/G1 phase (Fig. 6; $\mathrm{P}=0.036$ ).

Following $\mathrm{H}_{2} \mathrm{O}_{2}$ treatment, the number of apoptotic cells did not markedly increase, however, the hucMSCs became larger and flatter. Staining of hucMSCs with SA- $\beta$-gal indicated that hucMSCs were subjected to senescence following $\mathrm{H}_{2} \mathrm{O}_{2}$ stimulation (Fig. 6B). In the five $\mathrm{H}_{2} \mathrm{O}_{2}$-treated groups, the proportion of premature senescence cells, as measured by SA- $\beta$-gal 
A
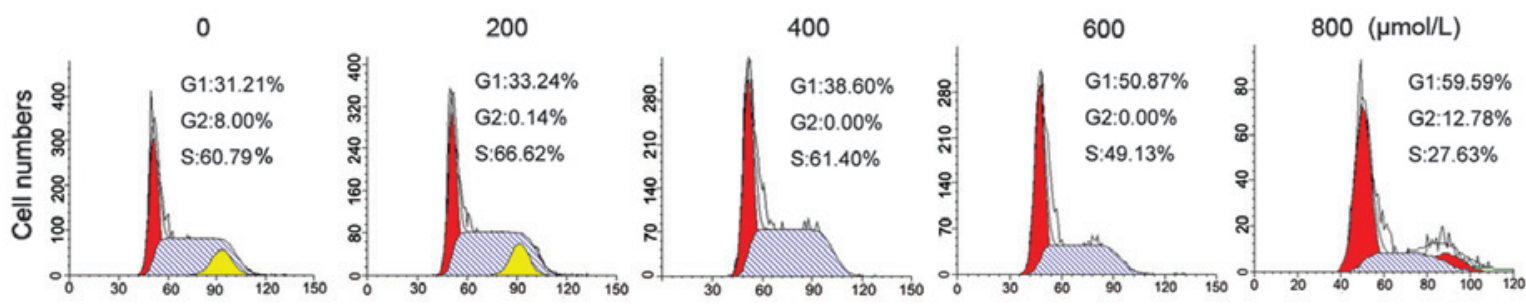

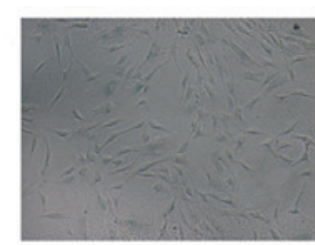

0

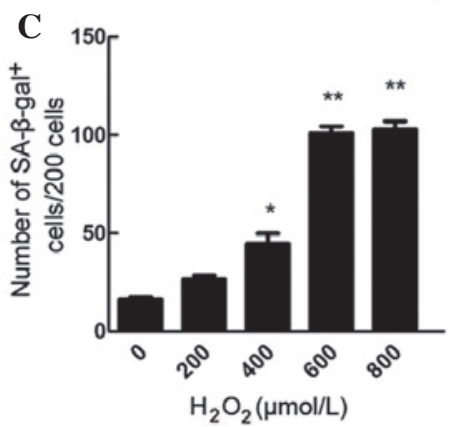

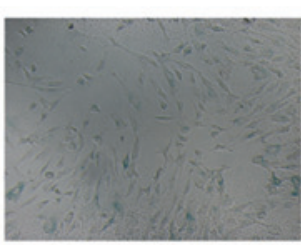

200

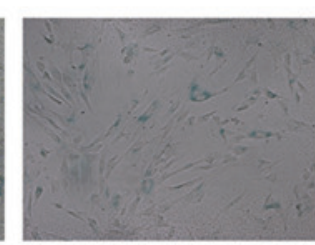

400

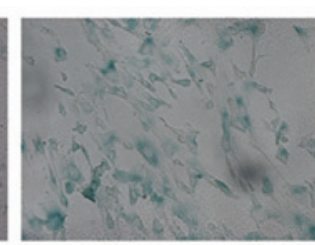

600

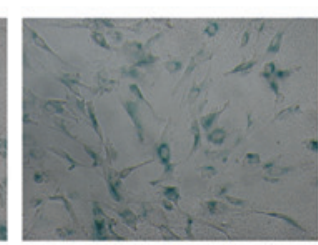

$800(\mu \mathrm{mol} / \mathrm{L})$
D
APE1/Ref-1

APE1/Ref-

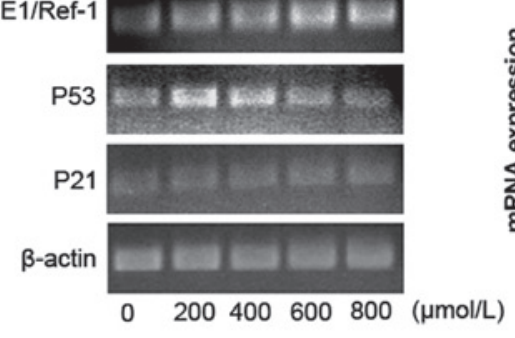

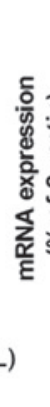

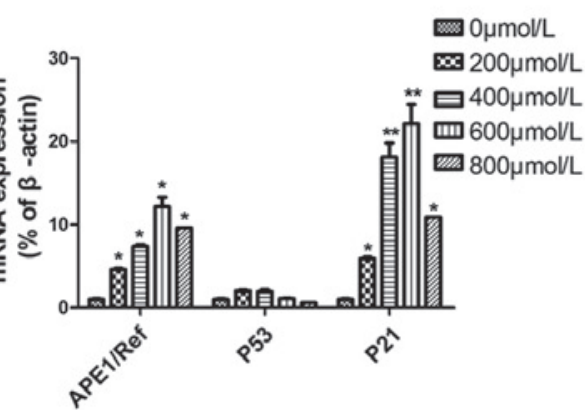

Figure 6. $\mathrm{H}_{2} \mathrm{O}_{2}$-induced cellular premature senescence in human umbilical cord mesenchymal stem cells (hucMSCs). (A) Cell cycle arrest in G1 phase following stimulation with different concentrations $\mathrm{H}_{2} \mathrm{O}_{2}$. (B) Staining of premature senescent hucMSCs with SA- $\beta$-gal under different $\mathrm{H}_{2} \mathrm{O}_{2}$ concentrations (magnification, x100). (C) Quantification of premature senescence cells. Cells stained blue (SA- $\beta$-gal-positive) indicated premature senescence hucMSCs. (D) mRNA expression level of APE1/Ref-1, P53 and P21 in premature senescent hucMSCs. ${ }^{*} \mathrm{P}<0.05,{ }^{* *} \mathrm{P}<0.01$ vs. $0 \mu \mathrm{mol} / 1$ treatment. SA- $\beta$-gal, senescence-associated $\beta$-galactosidase; APE1/Ref-1, apurinic/apyrimidinic endonuclease 1/redox factor-1.

staining, increased progressively with increasing concentrations of $\mathrm{H}_{2} \mathrm{O}_{2}(8.17,13.33,22.33,52.17$ and 50\%; Fig. 6C). This indicates that $\mathrm{H}_{2} \mathrm{O}_{2}$ promotes senescence of early passage hucMSCs in a concentration-dependent manner. However, the dose of $800 \mu \mathrm{M} \mathrm{H}_{2} \mathrm{O}_{2}$ exhibited a slight cytotoxic effect, with viable cells exhibiting poor adherence when washed with PBS. Hence, we propose that a dose of $600 \mu \mathrm{M} \mathrm{H}_{2} \mathrm{O}_{2}$ is suitable to establish a model of aging in hucMSCs. Additionally, treatment of $\mathrm{P} 4$ hucMSCs with $600 \mu \mathrm{M} \mathrm{H}_{2} \mathrm{O}_{2}$ caused them to become larger and flatter, and the cells appeared to be morphologically indistinguishable from replicative senescent cells (P11 or P17).

The expression levels of P53, P21 and APE1/Ref-1 are upregulated in replicative senescence and premature senescence. P53 $(\mathrm{P}>0.05)$ and P21 $(\mathrm{P}<0.05)$ mRNA expression levels were also increased following $\mathrm{H}_{2} \mathrm{O}_{2}$ treatment (Fig. 6D). However, at the higher concentrations of $\mathrm{H}_{2} \mathrm{O}_{2}(600$ and $800 \mu \mathrm{M}$ ), the expression level of P53 was reduced. The current study interpreted that excessive stimulation of oxidation may decrease the activities of P53 or even inactivate it. Additionally, exposure to $\mathrm{H}_{2} \mathrm{O}_{2}$ for $2 \mathrm{~h}$ resulted in dose-dependent increased expression levels of APE1/Ref-1 and P21 mRNA in the premature senescence hucMSCs $(\mathrm{P}<0.05$; Fig. 6D). This indicates that oxidative stress is important in regulating MSC senescence.

\section{Discussion}

Human MSCs have been isolated from various tissues and are a potential stem cell source for use in regenerative medicine.
Typically, stem cell numbers are limited and it is necessary to expand their populations in vitro prior to clinical use. In order to examine the characteristics and safety of long-term cultured hucMSCs, the current study evaluated the effects of long-term culture on hucMSC proliferation, phenotype, differentiation, intracellular ROS levels, cell cycle status and senescence-associated gene expression levels. In addition, with the aim to highlight the mechanisms that may cause oxidative stress and promote senescence, the present study established a cell model of $\mathrm{H}_{2} \mathrm{O}_{2}$-induced hucMSC premature senescence to analyze the associations between changes in the microenvironment and the process of aging $(15,25)$.

The present results indicate that hucMSCs undergo replicative senescence during long-term culture in vitro, as demonstrated by the altered cell proliferation curve, peculiar cell morphology, cell cycle arrest in G0/G1 and increased SA- $\beta$-gal activity, which are known markers of senescence. The current study observed that senescence occurred following a cumulative number of passages, ranging between $\mathrm{P} 4$ and P17. Additionally, hucMSCs expanded continuously for $\leq 30$ days and maintained the normal spindle shape under the culture conditions, without demonstrating increased SA- $\beta$-gal activity. Consistent with these findings, SAHF were observed in the late phases of hucMSC culture. SAHF is a specific heterochromatic structure accumulating in the nucleus in the form of punctate foci $(26,27)$. By contrast, the chromatin distribution during the early phases of culture was homogeneous. The middle phase may be an interim period of cellular senescence. 
Numerous studies have reported the differentiation of MSCs upon replicative senescence (28-31), however, the underlying regulatory mechanism is still controversial. Wagner et al (30) demonstrated that the propensity for osteogenic differentiation of MSCs increased and adipogenic differentiation potential decreased during in vitro senescence. Kim et al (28) reported that the adipogenic and osteogenic differentiation capacity of MSCs were decreased at $>30$ population doublings.

Oxidative stress is one of the major factors that accelerates cell senescence in vivo and in vitro $(32,33)$. The current study observed that the generation of ROS in hucMSCs increased during long-term in vitro culture. The environment in which cellular senescence evolved is replete with extrinsic hazards and the pace of senescence may be affected by the culture conditions $(10,34) . \mathrm{H}_{2} \mathrm{O}_{2}$ treatment of early phase hucMSCs provides a useful experimental model to analyze the mechanisms of senescence-associated changes (35-37). The results of the current study demonstrate that exogenous stress or oxidative damage cause changes in gene expression levels, contributing to certain changes observed in replicative senescence. It was also observed that the accumulation of intracellular ROS in senescent hucMSCs was accompanied by the upregulation of P21 and P53 mRNA levels. APE1/Ref-1 is able to activate transcription factors associated with the cellular response to various stresses against oxidative DNA damage (38). Similarly, in the present study, the mRNA expression levels of APE1/Ref-1 were elevated in senescent hucMSCs. These results indicate that the P53/P21 pathway may be the primary mediator of hucMSCs aging, and APE1/Ref-1 may be important in the processes of replicative and premature senescence.

In conclusion, the data indicate that long-term in vitro culture and extrinsic control, particularly oxidative stress pressure, are crucial in the regulation of stem cell aging. Thus, the quality of MSCs preparations should be carefully controlled prior to clinical application. Further research will be necessary to understand the mechanisms that regulate the replicative senescence of stem cells and how to ensure they remain in the early, normal phase during long-term culture.

\section{Acknowledgements}

This work was supported by the National Natural Science Foundation of China (grant no. 30840053), the Natural Science Foundation of Jiangsu Province (grant no. BK2008232) and the Foundation of the Jiangsu University for Senior Talented Investigator (grant no. 11JDG0089).

\section{References}

1. Forostyak S, Jendelova P and Sykova E: The role of mesenchymal stromal cells in spinal cord injury, regenerative medicine and possible clinical applications. Biochimie 95: 2257-2270, 2013.

2. Li T, Yan Y, Wang B, Qian H, Zhang X, Shen L, Wang M, Zhou Y, Zhu W, Li W and Xu W: Exosomes derived from human umbilical cord mesenchymal stem cells alleviate liver fibrosis. Stem Cells Dev 22: 845-854, 2013.

3. Zhang Y, Cai W, Huang Q, Gu Y, Shi Y, Huang J, Zhao F, Liu Q, Wei X, Jin M, et al: Mesenchymal stem cells alleviate bacteria-induced liver injury in mice by inducing regulatory dendritic cells. Hepatology 59: 671-682, 2014.

4. Woodbury D, Reynolds K and Black IB: Adult bone marrow stromal stem cells express germline, ectodermal, endodermal, and mesodermal genes prior to neurogenesis. J Neurosci Res 69: 908-917, 2002
5. Choudhery MS, Badowski M, Muise A and Harris DT: Comparison of human mesenchymal stem cells derived from adipose and cord tissue. Cytotherapy 15: 330-343, 2013.

6. Mamidi MK, Nathan KG, Singh G, Thrichelvam ST, Mohd Yusof NA, Fakharuzi NA, Zakaria Z, Bhonde R, Das AK and Majumdar AS: Comparative cellular and molecular analyses of pooled bone marrow multipotent mesenchymal stromal cells during continuous passaging and after successive cryopreservation. J Cell Biochem 113: 3153-3164, 2012.

7. Izadpanah R, Kaushal D, Kriedt C, Tsien F, Patel B, Dufour J and Bunnell BA: Long-term in vitro expansion alters the biology of adult mesenchymal stem cells. Cancer Res 68: 4229-4238, 2008.

8. Wagner W, Ho AD and Zenke M: Different facets of aging in human mesenchymal stem cells. Tissue Eng Part B Rev 16: 445-453, 2010.

9. Courtois-Cox S, Jones SL and Cichowski K: Many roads lead to oncogene-induced senescence. Oncogene 27: 2801-2809, 2008.

10. Chakkalakal JV, Jones KM, Basson MA and Brack AS: The aged niche disrupts muscle stem cell quiescence. Natrue 490: 355-360, 2012.

11. Brandl A, Meyer M, Bechmann V, Nerlich M and Angele P: Oxidative stress induces senescence in human mesenchymal stem cells. Exp Cell Res 317: 1541-1547, 2011.

12. Chandler $H$ and Peters G: Stressing the cell cycle in senescence and aging. Curr Opin Cell Biol 25: 765-771, 2013.

13. Kim YJ, Hwang SH, Lee SY, Shin KK, Cho HH, Bae YC and Jung JS: miR-486-5p induces replicative senescence of human adipose tissue-derived mesenchymal stem cells and its expression is controlled by high glucose. Stem Cells Dev 21: 1749-1760, 2012.

14. Zhang DY, Wang HJ and Tan YZ: Wnt/ $\beta$-catenin signaling induces the aging of mesenchymal stem cells through the DNA damage response and the p53/p21 pathway. PLoS One 6: e21397, 2011.

15. Ho PJ, Yen ML, Tang BC, Chen CT and Yen BL: H2O2 accumulation mediates differentiation capacity alteration, but not proliferative decline, in senescent human fetal mesenchymal stem cells. Antioxid Redox Signal 18: 1895-1905, 2013.

16. Zhou BR, Xu Y, Wu D, Permatasari F, Gao YY and Luo D: Ginsenoside Rg1 protects human fibroblasts against psoralenand UVA-induced premature senescence through a telomeric mechanism. Arch Dermatol Res 304: 223-228, 2012.

17. Thakur S, Sarkar B, Cholia RP, Gautam N, Dhiman M and Mantha AK: APE1/Ref-1 as an emerging therapeutic target for various human diseases: Phytochemical modulation of its functions. Exp Mol Med 46: e106, 2014.

18. Ju Z, Choudhury AR and Rudolph KL: A dual role of p21 in stem cell aging. Ann N Y Acad Sci 100: 333-344, 2007.

19. Herbig U, Jobling WA, Chen BP, Chen DJ and Sedivy JM: Telomere shortening triggers senescence of human cells through a pathway involving ATM, p53, and p21(CIP1), but not p16(INK4a). MOL CELL 14: 501-513, 2004.

20. Collado M, Blasco MA and Serrano M: Cellular senescence in cancer and aging. Cell 130: 223-233, 2007.

21. Kawasaki H, Guan J and Tamama K: Hydrogen gas treatment prolongs replicative lifespan of bone marrow multipotential stromal cells in vitro while preserving differentiation and paracrine potentials. Biochem Biophys Res Commun 397: 608-613, 2010.

22. Hao H, Chen G, Liu J, Ti D, Zhao Y, Xu S, Fu X and Han W: Culturing on Wharton's jelly extract delays mesenchymal stem cell senescence through p53 and p16INK4a/pRb pathways. PLoS One 8: e58314, 2013.

23. Lin TM, Tsai JL, Lin SD, Lai CS and Chang CC: Accelerated growth and prolonged lifespan of adipose tissue-derived human mesenchymal stem cells in a medium using reduced calcium and antioxidants. Stem Cells Dev 14: 92-102, 2005.

24. Sharpless NE and DePinho RA: How stem cells age and why this makes us grow old. Nat Rev Mol Cell Biol 8: 703-713, 2007.

25. Wang TT, Zeng GC, Li XC and Zeng HP: In vitro studies on the antioxidant and protective effect of 2-substituted -8-hydroxyquinoline derivatives against $\mathrm{H}_{2} \mathrm{O}_{2}$-induced oxidative stress in BMSCs. Chem Biol Drugs Des 75: 214-222, 2010.

26. Schellenberg A, Lin Q, Schuler H, Schüler H, Koch CM, Joussen S, Denecke B, Walenda G, Pallua N, Suschek CV, Zenke $M$ and Wagner W: Replicative senescence of mesenchymal stem cells causes DNA-methylation changes which correlate with repressive histone marks. Aging (Albany NY) 3: 873-888, 2011. 
27. Zhang R, Poustovoitov MV, Ye X, Santos HA, Chen W, Daganzo SM, Erzberger JP, Serebriiskii IG, Canutescu AA, Dunbrack RL, et al: Formation of MacroH2A-containing senescence-associated heterochromatin foci and senescence driven by ASF1a and HIRA. Dev Cell 8: 19-30, 2005.

28. Kim J, Kang JW, Park JH, Choi Y, Choi KS, Park KD, Baek DH, Seong SK, Min HK and Kim HS: Biological characterization of long-term cultured human mesenchymal stem cells. Arch Pharm Res 32: 117-126, 2009.

29. Li Z, Liu C, Xie Z, Song P, Zhao RC, Guo L, Liu Z and Wu Y: Epigenetic dysregulation in mesenchymal stem cell aging and spontaneous differentiation. Plos One 6: e20526, 2011.

30. Wagner W, Horn P, Castoldi M, Diehlmann A, Bork S, Saffrich R, Benes V, Blake J, Pfister S, Eckstein V and Ho AD: Replicative senescence of mesenchymal stem cells: a continuous and organized process. PLoS One 3: e2213, 2008.

31. Moerman EJ, Teng K, Lipschitz DA and Lecka-Czernik B: Aging activates adipogenic and suppresses osteogenic programs in mesenchymal marrow stroma/stem cells: The role of PPAR-gamma2 transcription factor and TGF-beta/BMP signaling pathways. Aging Cell 3: 379-389, 2004.

32. Edwards M, Rassin DK, Izumi T, Mitra S and Perez-Polo JR: APE/Ref-1 responses to oxidative stress in aged rats. J Neurosci Res 54: 635-638, 1998.
33. Tanaka T, Halicka HD, Huang $X$, Traganos $F$ and Darzynkiewicz Z: Constitutive histone H2AX phosphorylation and ATM activation, the reporters of DNA damage by endogenous oxidants. Cell Cycle 5: 1940-1945, 2006.

34. Wagner W, Horn P, Bork S and Ho AD: Aging of hematopoietic stem cells is regulated by the stem cell niche. Exp Gerontol 43: 974-980, 2008.

35. Yagi H, Tan J and Tuan RS: Polyphenols suppress hydrogen peroxide-induced oxidative stress in human bone-marrow derived mesenchymal stem cells. J Cell Biochem 114: 1163-1173, 2013.

36. Seo SK, Yang W, Park YM, Lee WT, Park KA and Lee JE: Overexpression of human arginine decarboxylase rescues human mesenchymal stem cells against $\mathrm{H}_{2} \mathrm{O}_{2}$ toxicity through cell survival protein activation. J Korean Med Sci 28: 366-373, 2013.

37. Wang FW, Wang Z, Zhang YM, Du ZX, Zhang XL, Liu Q, Guo YJ, Li XG and Hao AJ: Protective effect of melatonin on bone marrow mesenchymal stem cells against hydrogen peroxide-induced apoptosis in vitro. J Cell Biochem 114: 2346-2355, 2013.

38. Fritz G, Grösch S, Tomicic M and Kaina B: APE/Ref-1 and the mammalian response to genotoxic stress. Toxicology 193: 67-78, 2003. 